Journal of Computer Science 8 (9): 1514-1519, 2012

ISSN 1549-3636

(C) 2012 Science Publications

\title{
End to End Delay Improvement in Heterogeneous Multicast Network using Genetic Optimization
}

\author{
${ }^{1}$ V. Chandrasekar and ${ }^{2} \mathrm{~K}$. Baskaran \\ ${ }^{1}$ Department of CSE, \\ Dhanalakshmi Srinivasan Enginering College, Perambalur, 621212, India \\ ${ }^{2}$ Department of CSE, \\ Government College of Technology, Coimbatore, India
}

\begin{abstract}
Problem statement: Multicast is a concept of group communication which refers to transmitting the same data or messages from a source to multiple destinations in the network. This oneto-many group communication is a generalization of the concepts of one-to-one unicast and one-to-all broadcast. To deliver data from the sender to all receivers efficiently, routing plays an important role in multicast communication. In QoS multicast, every receiver must receive the data within their own specified QoS constraints. This becomes challenging especially if the network is a heterogeneous network made up of wired and wireless devices. Approach: This study investigates the performance of Protocol Independent Multicast-Sparse Mode (PIM-SM) protocol in a heterogeneous network running an video conferencing application and proposes an enhanced routing protocol using Genetic Optimizing techniques to improve QOS parameters in the wireless part. Results and Conclusion: Extensive simulations were carried out using the proposed technique and existing PIM-SM. The proposed optimization technique not only improves the throughput of the network but also decreased the end to end delay.
\end{abstract}

Key words: Protocol independent multicast-sparse mode, wireless network, heterogeneous network, genetic algorithm

\section{INTRODUCTION}

Audio video streaming data are popularly distributed to multiple hosts using multicast routing protocols. Multicasting sends data to multiple nodes in a group unlike either a unicast wherein a data packet has to be sent repeatedly to different nodes or broadcast wherein all the nodes in the network receive the data packet (Al-Hunaity et al., 2007). Multicast groups are formed by set of nodes interested in the same data and are represented by an IP address (Ballardie, 1997). The source node sends data to multicast group using the IP address. Routers between the source and recipient nodes forward copies to recipient nodes in different paths. Multicasting is complicated due to the varying processing power and network access bandwidth of the nodes, more so in a heterogeneous network containing both wired and wireless devices.

A multicast session is established by creating a multicast tree through which the data is transmitted to the multicast group. Multicast routing algorithms are used for constructing the multicast tree. Quality of Service (QoS) requirements such as end-to-end delay, delay variation, loss, cost, throughput are to be satisfied in group applications for efficient working of the network (Chen and Nahrsted, 1998). As the multicast tress spans through the wired and wireless devices in heterogeneous networks, resources along the path may fail to guarantee the required QoS leading to failure of the multicast tree. For efficient multicast communication, it is required that the tree constructed satisfies the resource requirements (Wang and Hou, 2000). The goal of QoS is to provide certain level of predictability and control on the service. Delay, Jitter, Bandwidth and Reliability are commonly used parameters that measure the QoS.

Multicast trees are built using either the Source Based Algorithms (SBA) or Core Based Algorithms (CBA). SBA constructs tree from the source node and connects up with all the recipients whereas in CBA, a core node is selected which acts as the root for the multicast tree and a core-based tree is a shortest-path tree rooted at some core node. In SBA (Al-Talib et al., 2009), a global state is maintained by every node and is used for tree construction in multicast protocols such as Protocol Independent Mode Dense Mode (PIM-DM) and 
Multicast Open Shortest Past First (MOSPF). CBA is used in many-to-many transmissions and is used for tree construction in Core Based Tree (CBT) and Protocol Independent Multicast Sparse Mode (PIM-SM).

The most commonly used multicast protocol is the Protocol Independent Multicast (PIM) (Deering et al., 1996). PIM is a multicast routing architecture to establish trees to many sparsely represented groups. The PIM is suitable for large heterogenous networks as it is robust, flexible and scalable (Alfawaer et al., 2007). PIM is a collection of protocols optimized for different scenario. PIM Sparse mode (PIM-SM) and PIM Dense mode (PIM-DM) are the most commonly used multicast protocols (Estrin et al., 1996). PIM-SM uses both sourcebased tress and core-based trees whereas PIM-DM uses only source-based trees. PIM-SM is widely used in all type of networks and PIM-DM is used mainly in small domains. In PIM routing protocols, PIM join and prune messages are used to join and leave a multicast distribution tree.

Biswas and Izmailov (2000) proposed a PIM-SM based IP-multicast routing framework for delivering heterogeneous quality of service. Two tree construction algorithms: TIQM and NUQM were proposed. TIQM depends upon the full availability of tree-specific information about a multicast group whereas NUQM does not require any tree specific information. Pseudo-optimal QoS-constrained trees are computed using TIQM but faces control-scalability problem. NUQM overcomes the control-scalability problem by restricting the amount of information used during tree computation. A QoS-extended intra-domain PIM-SM framework was presented.

Gomez-Montalvo et al. (2009) introduced an ontology framework for automatic networked multimedia systems deployment. The proposed framework takes into consideration the configuration driven by the requirements and preferences of the users. Heterogeneous multimedia environments due to various languages, protocols and hardware make it difficult for automatic deployment and interoperability of networked multimedia systems leading to major incompatibility issues. A case study was used to demonstrate the advantages of using this framework.

Gupta and Srimani (2003) presented a distributed core selection and migration protocols for mobile ad hoc networks. Core selection in ad hoc network is expensive due to the dynamic topology. The proposed core location method is based on median node of the multicast tree instead of the median node of the entire network. The proposed adaptive distributed core selection and migration method uses the median of the tree as the centroid of that tree. The cost of multicast tree is computed as the sum of weights of all the links in the tree, which gives the total bandwidth required for multicasting a packet. the cost of shortest-path tree rooted at the tree median, CostTM, is compared with the cost of shortest-path tree rooted at the median of the graph, CostGM. The simulation results show that the ratio CostTM/CostGM lies between 0.8-1.2 for different multicast groups.

Tseng and Chen (2001) introduced a distributed candidate's selection protocol, named DSDMR, which is self adapting based on group density. The proposed method uses an adaptive two-direction join mechanism. This address the problem of poor scalability resulting from high control overhead, un-robustness for centralized group manager and longer than necessary join latency. The proposed method performs well both in densely populated and sparsely populated networks. Extensive simulation results show that DSDMR can create low cost tree close to optimal greedy strategy with very low control overhead and join latency.

In this study it is proposed to implement Genetic Optimization techniques to improve the QOS for heterogeneous environments consisting of wired and wireless nodes.

\section{MATERIALS AND METHODS}

Gradient search techniques are intended for local search, obtaining solutions around the region of its starting point (Maalla et al., 2009). Global search techniques obtain more optimal solution, though it is dependent on an ideal set of starting values. The Genetic Algorithm (GA) is a population-based optimization algorithm, built on the basis of biological evolution. Gene sets are replicated, varied and mutated during the process of natural evolution. Similarly, mechanisms such as selection, reproduction, mutation are used in GA to evolve better solutions. The solutions to the optimization problem represented in bit-strings are the population used in GA. Fitness functions are used to evaluate each solution. The initial population is evolved through to the next generation on application of operators such as selection, reproduction, crossover and mutation. Better solutions are produced during each generation. The evolution process continues forming new generations till an appropriate solution is reached or a specific number of generations have been obtained. The pseudo-algorithm of GA is as follows:

\section{BEGIN:}

1. Generation $\mathrm{t}=0$;

2. Initialize the population $\mathrm{P}(\mathrm{t})$;

3. While not Termination criterion do 
a. Evaluate the population $\mathrm{P}(\mathrm{t})$;

b. Next $\mathrm{P}^{\prime}(\mathrm{t})$ (by crossover/mutation) over $\mathrm{P}(\mathrm{t})$;

c. Evaluate $P^{\prime}(t)$;

d. $P(t+1)=$ select from $P^{\prime}(t)$;

e. Generation $\mathrm{t}=\mathrm{t}+1$;

4. End while.

For each $n_{i} \in N_{r}$ a routing table is created. The table contains information of the $\mathrm{R}$ shortest, $\mathrm{R}$ cheapest and $\mathrm{R}$ least used paths. $\mathrm{R}$ is a parameter of the algorithm. A chromosome is represented by a string of length $|\mathrm{Nr}|$ in which each element (gene) $g_{i}$ represents a path between source $s$ and destination $n_{i}$. A route is selected based on the following parameters.

Discard individuals: In P, there may be duplicated chromosomes. Thus, new randomly generated individuals replace duplicated chromosomes.

Evaluate individuals: Objective functions are used to evaluate the individuals of $\mathrm{P}$. Then, non-dominated individuals of $\mathrm{P}$ are compared to the individuals in Pnd to update the non-dominated set, removing from Pnd dominated individuals.
Compute fitness: SPEA procedure is used to compute the fitness of each individual.

Selection: A roulette selection operator is applied over the set $\mathrm{P}_{\mathrm{nd}} \mathrm{UP}$ to generate the next evolutionary population $\mathrm{P}$.

Crossover and mutation: In this work a two-point crossover operator over selected pair of individuals is proposed with some genes in each chromosome of the new population being randomly changed (mutated), obtaining a new solution. The process continues until a stop criterion or the given maximum number of generations, is satisfied.

\section{RESULTS AND DISCUSSION}

The proposed system was implemented as a layer over PIM-SM and tested in a heterogeneous environment. Figure 1 shows the experimental test bed used for simulation. The throughput obtained in the proposed system for video traffic and compared with PIM-SM is shown in Fig. 2-4 show the end to end delay and the overall data dropped in the network respectively.

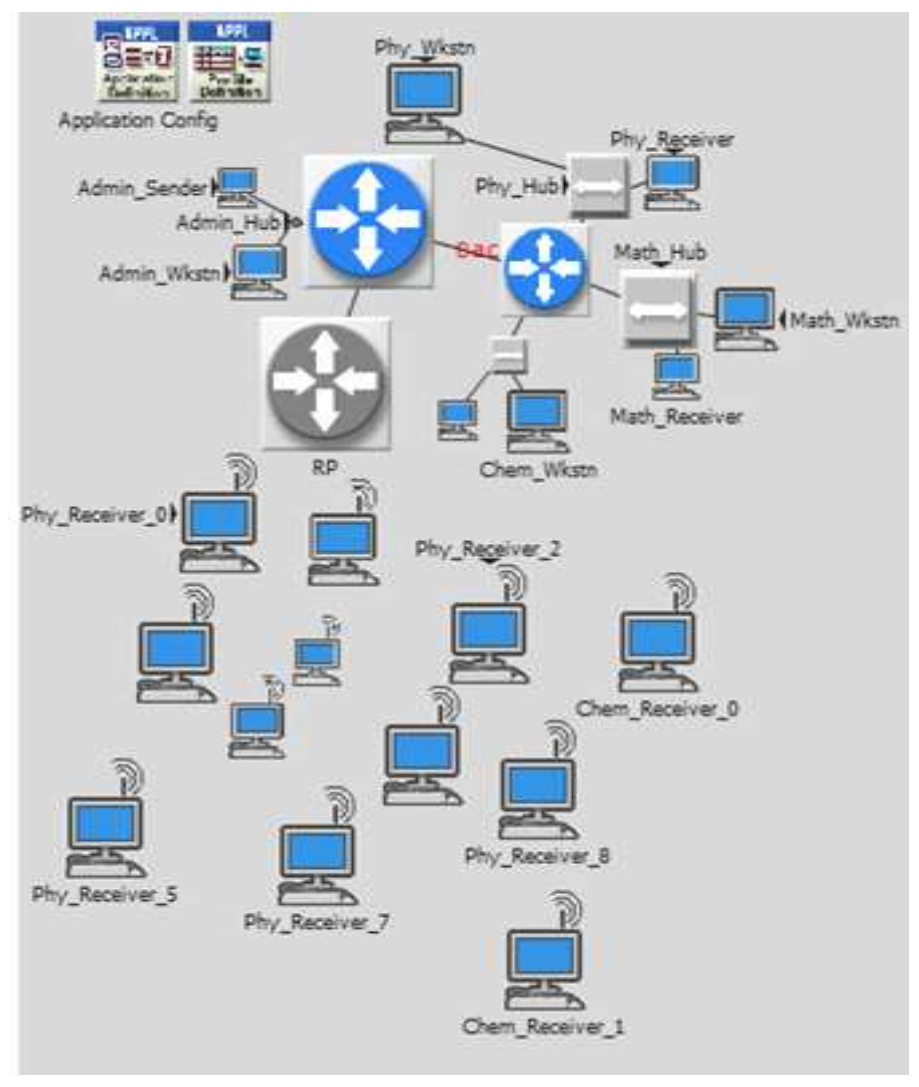

Fig. 1: The experimental test bed used in this study 


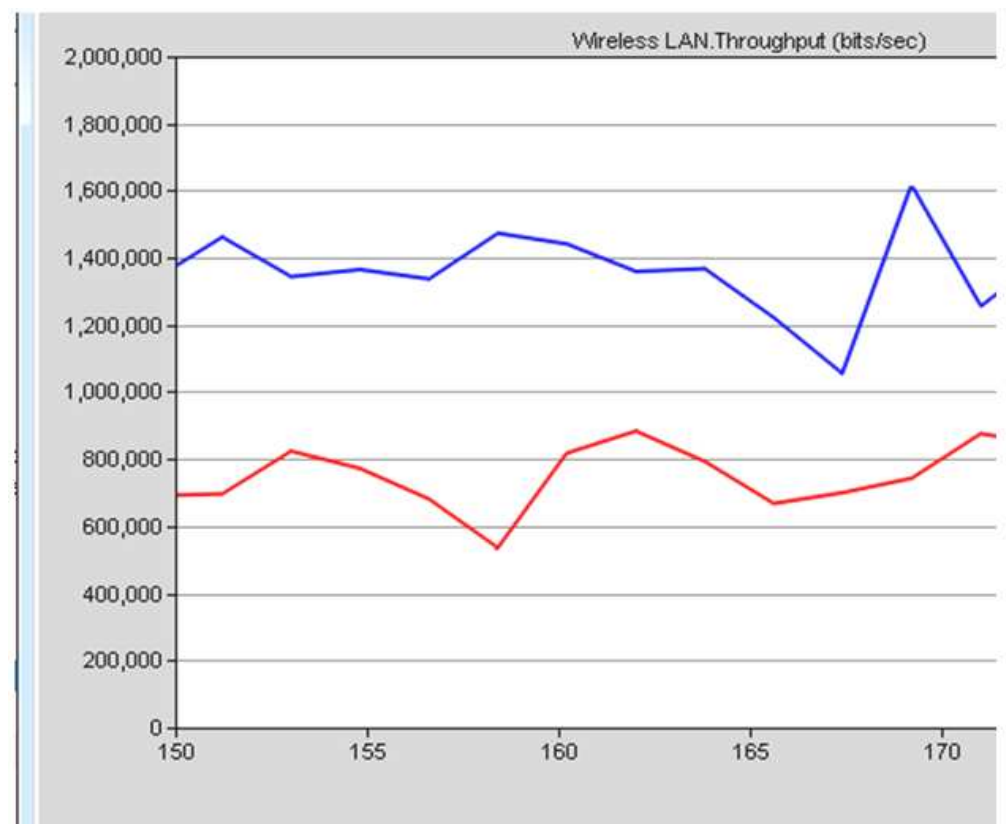

Fig. 2: The throughput obtained in the WLAN section

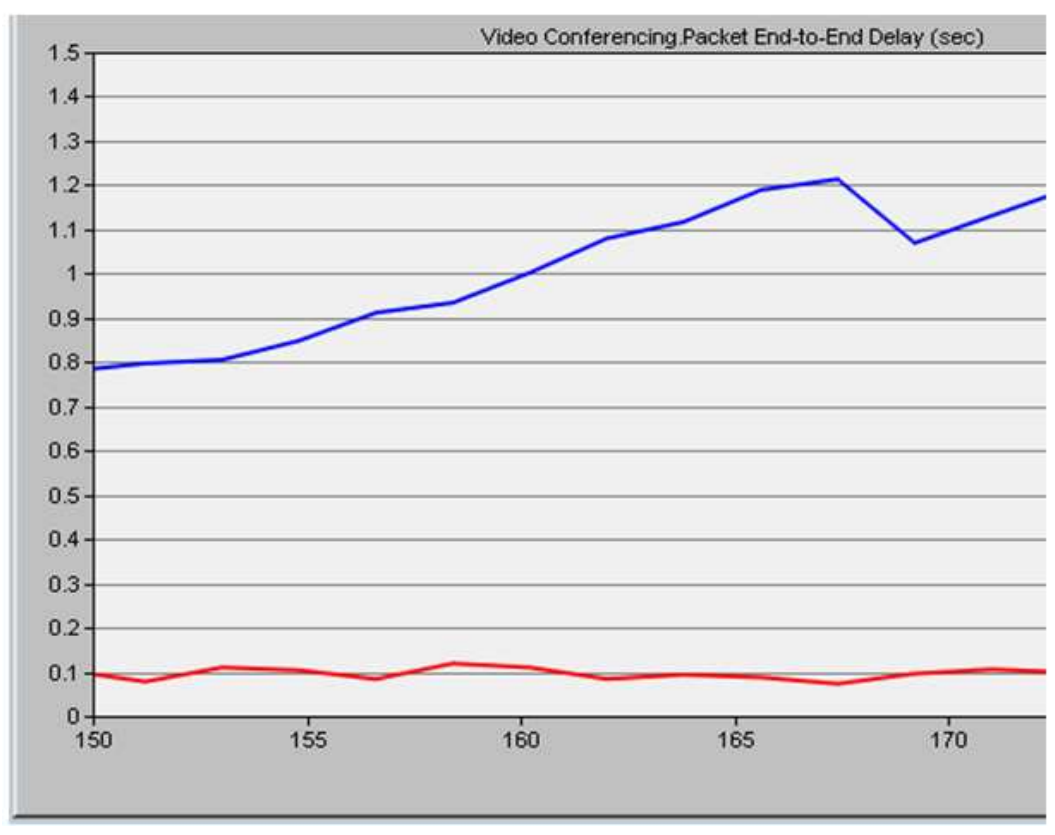

Fig. 3: The overall end to end delay in the heterogeneous network

Figure 2 shows the improvement of the throughput in the wireless section of the proposed optimization technique. It can be seen the proposed optimization technique (blue graph) increases the throughput by more than $75 \%$ for video transmission. Figure 3 shows the overall end to end delay in the network.
It can be seen that the end to end delay for the video conferencing increases, however the uniform delay between the wired and wireless nodes improves the Quality of service by reducing the jitter. The packet dropped in the wireless lan section drops considerably due to the genetic optimization as shown in Fig. 4. 


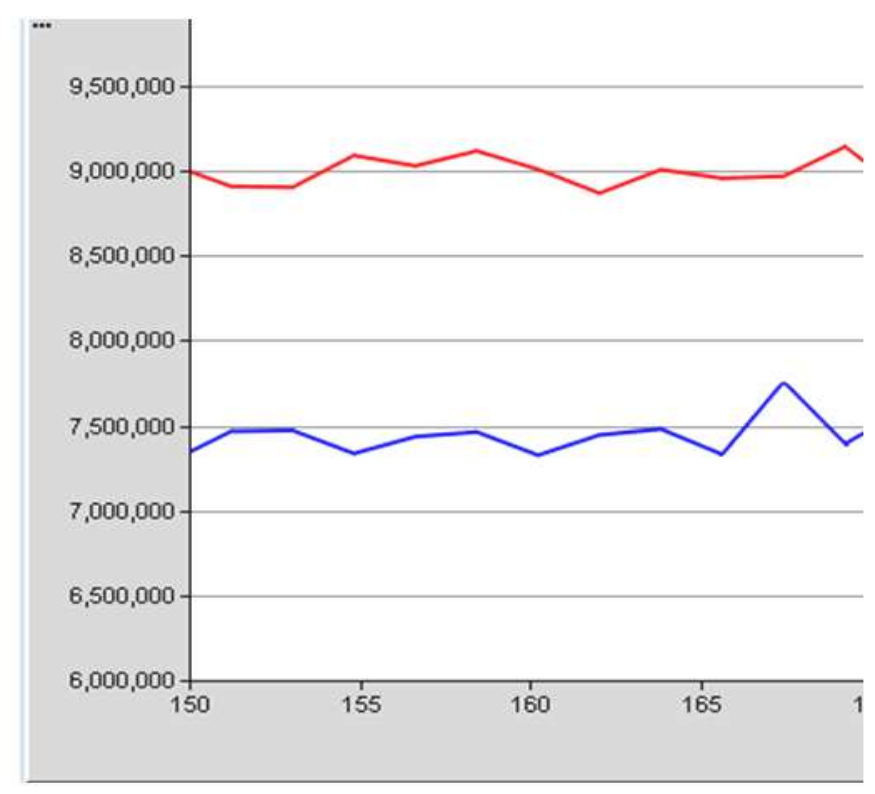

Fig. 4: The data dropped in bits/second

\section{CONCLUSION}

In this study it was proposed to investigate the effectiveness of PIM-SM in heterogeneous environment consisting of wireless and wired nodes. Video conferencing traffic was used in this study due to its critical QOS parameters. A novel optimization technique over the PIM-SM stack was proposed using genetic optimization. The proposed GO-PIM reduced the overall end to end delay in the network. While increasing the throughput of the network.

\section{REFERENCES}

Alfawaer, Z.M., G. Hua and N. Ahmed, 2007. A novel multicast routing protocol for mobile ad hoc networks. Am. J. Applied Sci., 4: 333-338. DOI: 10.3844/ajassp.2007.333.338

Al-Hunaity, M.F., S.A. Najim and I.M. El-Emary, 2007. A comparative study between various protocols of MANET networks. Am J. Applied Sci., $\quad 4:$ 663-665. DOI: 10.3844/ajassp.2007.663.665

Al-Talib, S.A., B.M. Ali and S. Khatun, 2009. An approach to improve the state scalability of source specific multicast. Am. J. Applied Sci., 6: 1347-1351.

DOI: 10.3844/ajassp.2009.1347.1351

Ballardie, A., 1997. Core Based Trees (CBT) multicast routing architecture.
Biswas, S.K. and R. Izmailov, 2000. A QoS-aware routing framework for PIM-SM based IP-multicast, Proceedings of the IEEE Global Telecommunications Conference, Nov. 27-Dec. 1, IEEE Xploer Press, San Francisco, CA., pp: 376381. DOI: 10.1109/GLOCOM.2000.892033

Chen, S. and K. Nahrsted, 1998. An overview of quality of service routing for next-generation high-speed networks: Problems and solutions. IEEE Netw., 12: 64-79. DOI: $10.1109 / 65.752646$

Deering, S., D.L. Estrin, D. Farinacci, V. Jacobson and C.G. Liu et al., 1996. The PIM architecture for wide-area multicast routing. IEEE/ACM Trans. Network., 4: 153-162.

Estrin, D., D. Farinacci, A. Helmy, D. Thaler and S. Deering et al., 1996. Protocol Independent Multicast-Sparse Mode (PIM-SM): Protocol specification. University of Southern California.

Gomez-Montalvo, J., M. Lamolle and E. Exposito, 2009. A multimedia ontology driven architecture framework (MODA) for networked multimedia systems. Proceedings of the First International Conference on Networked Digital Technologies, Jul. 28-31, IEEE Xplore Press, Ostrava, pp: 411416. DOI: $10.1109 /$ NDT.2009.5272081

Gupta, S.K.S. and P.K. Srimani, 2003. Adaptive core selection and migration method for multicast routing in mobile ad hoc networks. IEEE Trans. Parallel Distrib. Syst., 14: 27-38. DOI: 10.1109/TPDS.2003.1167368 
Maalla, A., C. Wei and H.J. Taha, 2009. Optimal power multicast problem in wireless mesh networks by using a hybrid particle swarm optimization. Am. J. Applied Sci., 6: 1758-1762. DOI: 10.3844/ajassp.2009.1758.1762

Tseng, C.J. and C.H. Chen, 2001. The performance of QoS-aware IP multicast routing protocols. Networks, 42: 97-108. DOI: 10.1002/net.10084
Wang, B. and J.C. Hou, 2000. Multicast routing and its QoS extension: Problems, algorithms and protocols. IEEE Netw., 14: 22-36. DOI: $10.1109 / 65.819168$ 